\title{
Evaluation of electromotive force in interplanetary space
}

\author{
Yasuhito Narita $^{1,2}$ and Zoltán Vörös ${ }^{1,2,3}$ \\ ${ }^{1}$ Space Research Institute, Austrian Academy of Sciences, Schmiedlstr. 6, 8042 Graz, Austria \\ ${ }^{2}$ Institute of Physics, University of Graz, Universitätsplatz 5, 8010 Graz, Austria \\ ${ }^{3}$ Department of Geophysics and Space Sciences, Eötvös University, Budapest, Hungary
}

Correspondence: Yasuhito Narita (yasuhito.narita@oeaw.ac.at)

Received: 29 October 2017 - Revised: 19 December 2017 - Accepted: 19 December 2017 - Published: 24 January 2018

\begin{abstract}
Electromotive force plays a central role in the turbulent dynamo mechanism and carries important information on the nature of the turbulent fields. In this study, an analysis method is developed for the electromotive force and the transport coefficients such as those for the $\alpha$ effect (coefficient $\alpha$ ) and the turbulent diffusivity (coefficient $\beta$ ). The method is applied to a magnetic cloud event observed by the Helios 2 spacecraft in the inner heliosphere. The electromotive force is enhanced together with the magnetic cloud event by 1 or 2 orders of magnitude, suggesting that the magnetic field can locally be amplified in the heliosphere, presumably for a short time.
\end{abstract}

Keywords. Interplanetary physics (interplanetary magnetic fields) - space plasma physics (transport processes)

\section{Introduction}

The essential part in the dynamo mechanism amplifying the large-scale magnetic field lies in the existence of electromotive force. In the theory of mean-field electrodynamics, the electromotive force is defined as a statistically averaged vectorial quantity, and it is a cross product between the fluctuating flow velocity $\delta \boldsymbol{U}$ and the fluctuating magnetic field $\delta \boldsymbol{B}$,

$\mathcal{E}_{\mathrm{em}}=\langle\delta \boldsymbol{U} \times \delta \boldsymbol{B}\rangle$.

Here, the angular brackets $\langle\cdots\rangle$ denote the operation of ensemble averaging over many realizations. The electromotive force has dimensions of the electric field.

In the mean-field dynamo theory, the electromotive force is assumed to be linear in the mean magnetic field $\boldsymbol{B}_{0}$ to the first order, and also in the spatial gradient of the mean field such as the curl of the mean magnetic field, $\nabla \times \boldsymbol{B}_{0}$ (one may also use the current density $\boldsymbol{j}$ using Ampère's law), and the curl of the mean flow velocity, $\nabla \times \boldsymbol{U}_{0}$ (which is the vorticity). A more comprehensive form for the electromotive force using up to the first-order derivatives of the mean fields is expressed as (Yokoi, 2013)

$\mathcal{E}_{\mathrm{em}}=\alpha \boldsymbol{B}_{0}-\beta \nabla \times \boldsymbol{B}_{0}+\gamma \nabla \times \boldsymbol{U}_{0}$,

where the first term on the right-hand side in Eq. (2) with the coefficient $\alpha$ and the mean magnetic field serves as the amplification of the mean magnetic field by small-scale twisting flow motions. The second term with the coefficient $\beta$ (which has the same dimension as that of the magnetic diffusivity) and the curl of the mean magnetic field (which is proportional to the electric current density for the mean magnetic field) is the turbulent magnetic diffusion, and the third term with the coefficient $\gamma$ and the curl of the large-scale flow velocity the cross helicity dynamo.

A simpler form or a minimal expression of the electromotive force for a dynamo mechanism is composed of the two terms associated with the mean magnetic field only (Parker, 1955; Steenbeck et al., 1966; Steenbeck and Krause, 1966):

$\mathcal{E}_{\mathrm{em}}=\alpha \boldsymbol{B}_{0}-\beta \nabla \times \boldsymbol{B}_{0}$.

Equation (3) was historically proposed before the importance of the cross helicity term was recognized in studies on the sunspot number variation and the accretion disk dynamo (Yoshizawa, 1990; Yoshizawa and Yokoi, 1993; Yokoi, 1996, 1999). Here again, the first term with $\alpha$ is responsible for the growth of the mean magnetic field, and it is essential to the $\alpha$ effect due to the amplification of the magnetic field by a turbulent twisted flow motion. The second term with $\beta$ is destructive and contributes to an effective diffusion of the large-scale fields when coupled to the induction equation (turbulent diffusion). Equation (3) is obtained from the 
induction equation for the fluctuating magnetic field using the first-order smoothing approximation (see, e.g., Choudhuri, 1998, for a concise derivation). The coefficients $\alpha$ and $\beta$ are in fact tensors (of rank 2 and 3, respectively). In the isotropic turbulence treatment, these tensors reduce to scalars (the $\alpha$ tensor becomes diagonal and the $\beta$ tensor is proportional to the Levi-Civita antisymmetric tensor). In our work, we use the simpler form (Eq. 3) for the sake of brevity in mathematical operations in the spirit of proof of concept.

It is important to note that the electromotive force is a second-order fluctuation quantity and is in the same class as the energy densities of the fluctuating fields (for the magnetic field and the flow velocity) and the helicity densities (magnetic helicity, kinetic helicity, and cross helicity). The electromotive force appears as off-diagonal elements of the covariance matrix composed of the fluctuating magnetic field and fluctuating velocity (Narita, 2017).

Here we propose an analysis method to determine the electromotive force using in situ spacecraft data in space plasma. While a large number of studies on turbulent space plasmas (e.g., solar wind turbulence) concentrate on the behavior of the energy and helicity quantities (see, e.g., reviews and monographs such as Tu and Marsch, 1995; Petrosyan et al., 2010; Bruno and Carbone, 2013), only few observational studies have been performed related to the discussion on the electromotive force in space plasma physics, e.g., an impulsive solar wind event in the Earth magnetosphere (Lundin et al., 2003) and turbulent solar wind plasma (Marsch and $\mathrm{Tu}, 1992,1993)$. In particular, Marsch and Tu $(1992,1993)$ discovered evidence that the electromotive force follows a power-law spectrum in the solar wind (indicating turbulent electromotive force) and is not simply proportional in the mean magnetic field. The electromotive force is, in contrast to the limited space plasma studies, one of the primary study targets in the laboratory pinch plasmas (Fontana et al., 2000; Ji and Prager, 2002) and in the liquid sodium experiment (Rahbarnia et al., 2012). This paper is motivated by a wish to fill the gap in the application of the electromotive force between laboratory and space plasma studies.

\section{Estimates of transport coefficients}

When modeling with $\boldsymbol{B}_{0}$ and $\nabla \times \boldsymbol{B}_{0}$ and neglecting the other terms or contributions from the cross helicity or the higherorder derivatives of the mean fields, it is possible to evaluate the transport coefficients $\alpha$ and $\beta$ directly from the measurement of the electromotive force. To this goal, we first build a vector product of the mean-field model of the electromotive force (Eq. 3) with the mean magnetic field $\boldsymbol{B}_{0}$ and eliminate the term with the coefficient $\alpha$. We obtain an estimator for the coefficient $\beta$ after some vector calculus:

$\beta_{\mathrm{obs}}=-\frac{1}{H^{2}} \boldsymbol{H} \cdot\left(\boldsymbol{B}_{0} \times \mathcal{E}_{\mathrm{em}}\right)$, where

$\boldsymbol{H}=\boldsymbol{B}_{0} \times\left(\nabla \times \boldsymbol{B}_{0}\right)$

and $H=|\boldsymbol{H}|$. We now build a scalar product of Eq. (3) with the mean magnetic field $\boldsymbol{B}_{0}$. Since the coefficient $\beta$ is evaluated in Eq. (4), we obtain an estimator for the coefficient $\alpha$ :

$\alpha_{\mathrm{obs}}=\frac{1}{B_{0}^{2}} \boldsymbol{B}_{0} \cdot \mathcal{E}_{\mathrm{em}}+\frac{\beta}{B_{0}^{2}} \boldsymbol{B}_{0} \cdot\left(\nabla \times \boldsymbol{B}_{0}\right)$.

The inputs to the calculation of the coefficients $\alpha$ and $\beta$ are the electromotive force $\mathcal{E}_{\text {em }}$, the mean magnetic field $\boldsymbol{B}_{0}$, and the curl of the mean magnetic field $\nabla \times \boldsymbol{B}_{0}$. When the measurement is performed by a single-point sensor (or spacecraft) in a supersonic or super-Alfvénic flow, one may limit the gradient direction to the flow direction and introduce an assumption that the spatial derivative is mostly a time derivative with advection by the mean flow $\boldsymbol{U}_{0}$,

$\nabla \simeq-\boldsymbol{e}_{\mathrm{U}} \frac{1}{U_{0}} \frac{\partial}{\partial t}=-\frac{\boldsymbol{U}_{0}}{U_{0}^{2}} \frac{\partial}{\partial t}$,

where $U_{0}=\left|\boldsymbol{U}_{0}\right|$ is the absolute value of the flow speed and $\boldsymbol{e}_{\mathrm{U}}=\boldsymbol{U}_{0} / U_{0}$ the unit vector in the direction of the mean flow. Note that Eq. (7) assumes that the spatial gradient is parallel to the mean flow, which reduces the problem to one spatial dimension, and that the spatial derivative is estimated by the time derivative using Eq. (7), which is equivalent to the stationarity of the mean field, $\mathrm{d} / \mathrm{d} t=\partial / \partial t+\boldsymbol{U}_{0} \cdot \nabla=0$. In this paper, the time derivative is evaluated using the first-orderaccuracy backward difference due to the irregularly sampled time series data,

$$
\left.\frac{\partial \boldsymbol{B}_{0}}{\partial t}\right|_{t} \simeq \frac{\boldsymbol{B}_{0}(t)-\boldsymbol{B}_{0}(t-\Delta t)}{\Delta t},
$$

where $\Delta t$ is the time resolution in the data. Thus, the $x$ component of the curl of the mean magnetic field is evaluated as

$$
\begin{aligned}
{\left[\nabla \times \boldsymbol{B}_{0}\right]_{x} \simeq } & \frac{1}{U_{0}^{2}}\left[-U_{0 y} \frac{\partial B_{0 z}}{\partial t}+U_{0 \mathrm{z}} \frac{\partial B_{0 y}}{\partial t}\right] \\
\simeq & -\frac{U_{0 y}}{U_{0}^{2}} \frac{B_{0 z}(t)-B_{0 z}(t-\Delta t)}{\Delta t}+ \\
& \frac{U_{0 z}}{U_{0}^{2}} \frac{B_{0 y}(t)-B_{0 y}(t-\Delta t)}{\Delta t} .
\end{aligned}
$$

The $y$ and $z$ components of the curl of the mean magnetic field are obtained by circulating $\{x, y, z\}$ into $\{y, z, x\}$ and $\{z, x, y\}$ in Eqs. (9) or (10), respectively.

\section{Magnetic cloud in interplanetary space}

The estimator for the electromotive force and that for the transport coefficients ( $\alpha$ and $\beta$ ) are tested against a magnetic 
cloud event in interplanetary space using the magnetic field and plasma (ion) data from the Helios 2 spacecraft (Porsche, 1977). The magnetic field data are obtained by the fluxgate magnetometer (also referred to as the saturation core magnetometer or the Förstersonde magnetometer) (Musmann et al., 1975) and the plasma data by the electrostatic analyzer (Schwenn et al., 1975; Rosenbauer et al., 1977). Merged data between the magnetic field and the plasma measurements are used. The sampling rate varies from $40 \mathrm{~s}$ to multitudes of $40 \mathrm{~s}$, and there are data gaps as well. Figure 1 displays the magnetic field magnitude, the proton bulk speed, the proton number density, and the electromotive force magnitude as time series plots from 17 to 20 April 1978. The dots in black represent the original measurements, and the solid lines in gray represent the smoothed data. The Helios 2 spacecraft is in an inbound orbit in the inner heliosphere and moves from a heliocentric distance of $0.41 \mathrm{AU}$ (astronomical unit) on 17 April 1978 to a distance of 0.37 AU on 20 April 1978. A magnetic cloud passes by the spacecraft around 18:0020:00 UT on 18 April. The magnetic field magnitude increases from about $40 \mathrm{nT}$ to about $70 \mathrm{nT}$, the ion bulk speed from about 500 to $800 \mathrm{~km} \mathrm{~s}^{-1}$, and the ion number density from about 30 to about $300 \mathrm{~cm}^{-3}$.

The electromotive force $\mathcal{E}_{\mathrm{em}}$ is evaluated by constructing the mean field and the fluctuation field from the time series data. The detailed procedure is as follows.

1. Mean field determination. The mean field is determined by assuming the ergodic hypothesis and regarding the mean values in the time domain as statistically representative of ensemble average. We use a fixed time window of $3 \mathrm{~h}$ ( $90 \mathrm{~min}$ before the window center and after the center). The averaging period is determined as a compromise such that the fluctuation fields have zero mean values under the conditions of the shortest periods and a sufficient number of data points within the time window. In this work, the smoothing is computed typically over 130 to 270 data points, depending on data availability (due to changes in the sampling rate) within each fixed time window of $3 \mathrm{~h}$. The mean fields for the magnetic field, the flow velocity (for the protons), and the density (again for the protons) at the $m$ th time record $t_{m}$ are determined by the box-car averaging method using the number of data points $N$ within the time window, e.g., $\boldsymbol{B}_{0}\left(t_{m}\right)=\frac{1}{N} \sum_{n=0}^{N-1} \boldsymbol{B}\left(t_{m+n-N / 2}\right)$, for the magnetic field. Other possibilities of the mean field determination are discussed in the last section of the paper.

2. Fluctuation field determination. The fluctuation fields are obtained by subtracting the mean field from the measured field, e.g., $\delta \boldsymbol{B}\left(t_{m}\right)=\boldsymbol{B}\left(t_{m}\right)-\boldsymbol{B}_{0}\left(t_{m}\right)$, for the magnetic field.

3. Electromotive force. The electromotive force is determined in the time domain by building a cross product between the fluctuation flow velocity and the fluctuation magnetic field and then averaging over the same time window as that for the mean fields (again, using the boxcar averaging), $\mathcal{E}_{\mathrm{em}}\left(t_{m}\right)=\frac{1}{N} \sum_{n=0}^{N-1} \delta \boldsymbol{U}\left(t_{m+n-N / 2}\right) \times$ $\delta \boldsymbol{B}\left(t_{m+n-N / 2}\right)$.

The electromotive force increases from about $10^{2} \mathrm{mV} \mathrm{km}^{-1}$ before the magnetic cloud event to above $10^{3} \mathrm{mV} \mathrm{km}^{-1}$ during the magnetic cloud event. For reference, the order of the electromotive force is $1 \mathrm{mV} \mathrm{km}^{-1}$ for a flow velocity fluctuation of $1 \mathrm{~km} \mathrm{~s}^{-1}$ and a magnetic field fluctuation of $1 \mathrm{nT}$.

The transport coefficients $\alpha$ and $\beta$ are evaluated using Eqs. (6) and (4), respectively, and their magnitudes are displayed as a function of time in the bottom two panels in Fig. 1. The order of the coefficient $\alpha$ is $1 \mathrm{~km} \mathrm{~s}^{-1}$ for an electromotive force of $1 \mathrm{mV} \mathrm{km}^{-1}$ and a mean magnetic field of $1 \mathrm{nT}$, and that of $\beta$ is $1 \mathrm{~km}^{2} \mathrm{~s}^{-1}$ for an electromotive force of $1 \mathrm{mV} \mathrm{km}^{-1}$ and a curl of the magnetic field of $1 \mathrm{nT} \mathrm{km}^{-1}$.

The coefficient $\alpha$ fluctuates around $1 \mathrm{~km} \mathrm{~s}^{-1}$ overall with an excursion to about $10^{1}$ to $10^{2} \mathrm{~km} \mathrm{~s}^{-1}$ during the magnetic cloud event. If we use an estimate of $50 \mathrm{nT}$ for the mean magnetic field and $10^{3} \mathrm{mV} \mathrm{km}^{-1}$ for the electromotive force, we obtain the coefficient $\alpha$ at about $20 \mathrm{~km} \mathrm{~s}^{-1}$, which roughly agrees with the peak value of $\alpha$.

In contrast, the coefficient $\beta$ has by far larger values throughout the observed time interval and varies by about 4 orders of magnitude between $10^{10} \mathrm{~km}^{2} \mathrm{~s}^{-1}$ at the beginning of 17 April 1978 and a peak of nearly $10^{14} \mathrm{~km}^{2} \mathrm{~s}^{-1}$ around 19:00 UT on 19 April 1978 at the time of magnetic cloud passing. It is interesting to observe that the enhancement of the coefficient $\beta$ is found not only during the magnetic cloud event but also during other periods, for example, around 02:00-03:00 UT on 18 April 1978 when the magnetic field magnitude is at a local minimum. Another interesting feature is that the variation sense of the coefficient $\beta$ changes from an anticorrelation sense to that of the coefficient $\alpha$ (e.g., 00:0006:00 UT on 17 April 1978) into a positive correlation sense (entire period on 18 April 1978) and then back into the anticorrelation sense (06:00-18:00 UT on 19 April 1978). Since the coefficient $\beta$ is an index of turbulent magnetic diffusion, the variation profile of $\beta$ indicates that turbulence occurs in an inhomogeneous way in the solar wind; some variations are accompanied by the magnetic cloud, others not. A naive estimate for the reason of the large values of the coefficient $\beta$ is as follows. We use values of $10^{2} \mathrm{mV} \mathrm{km}^{-1}$ for the electromotive force and $10^{-5} \mathrm{nT} \mathrm{km}^{-1}$ (which is $10^{-17} \mathrm{~V} \mathrm{~s} \mathrm{~m}^{-3}$ ) for the curl of the mean magnetic field and obtain a value of the coefficient $\beta$ about $10^{7} \mathrm{~km}^{2} \mathrm{~s}^{-1}$, which is the lower limit of the measured profile for the coefficient $\beta$. Here we used a flow speed of $500 \mathrm{~km} \mathrm{~s}^{-1}$ and a varying timescale of $1000 \mathrm{~s}$ for the advection and a magnetic field of $10 \mathrm{nT}$.

A close inspection shows that the curl of the mean magnetic field is even much smaller than the estimate above and is about $10^{-10} \mathrm{nT} \mathrm{km}^{-1}$, which gives the coefficient $\beta$ of the order of $10^{12} \mathrm{~km}^{2} \mathrm{~s}^{-1}$. There are various contributions that 
suppress the values of the curl of the mean field. The assumption of the one-dimensional advected structure is motivated by the use of single spacecraft data. On the one hand, the alignment of the mean magnetic field with its curl implies the use of a force-free field or a field-aligned current configuration, realized in various space plasma environments. The alignment may have a more fundamental nature. On the other hand, the constraint to the one-dimensional advected structure in the data analysis can be tested against multi-point measurements, from Cluster (Escoubet et al., 2001) or Magnetospheric Multiscale (MMS) (Burch et al., 2016). For example, it would be interesting to compare two different scenarios upon the direction of the spatial gradient using the multi-point data: first, if the gradient of the large-scale fields is mostly aligned with the mean flow direction and, second, if the gradient is perpendicular to the mean magnetic field direction as is the case for solar wind turbulence on ion kinetic scales (at about $400 \mathrm{~km}$ ) (Perschke et al., 2014). Perhaps the alignment may be scale-dependent from the fluid picture of plasma (on the length scales of the order of $10000 \mathrm{~km}$ ) down to ion-kinetic scale (of the order of $400 \mathrm{~km}$ ), and the smallscale field-aligned currents may be an important component to the turbulent solar wind. Also, continuous measurements at a higher sampling rate are available with the Cluster and MMS missions in comparison to the plasma measurements by the Helios spacecraft limited to $40 \mathrm{~s}$.

\section{Outlook}

The electromotive force is a second-order quantity such as the energy densities (magnetic energy and kinetic energy) and the helicity densities (magnetic helicity, kinetic helicity, and cross helicity) of the fluctuating fields, but its analysis using the in situ spacecraft data in space plasma has largely been overlooked in earlier studies. Although assumptions have to be incorporated, such as the use of one-dimensional advected structure in the time series data, it is possible to observationally evaluate the electromotive force and determine the transport coefficients using the mean field model for the dynamo theory. Studies on the transport coefficients in the turbulence and dynamo theories can be performed not only by the analytic or numerical methods but also by the observational method. The advantage of the presented method is that even data with different sampling rates can be used to the studies of the electromagnetic force and the transport coefficients at the cost of first-order accuracy approximation in the gradient computation.

Although the proposed method is rather a simple or a naive one, the analysis shows an enhancement of the electromotive force at the magnetic cloud event by 1 or 2 orders of magnitude. This result indicates a scenario that the enhanced or strong magnetic fields in the heliosphere are not merely generated in the Sun or in the solar atmosphere and stream into the heliosphere, but they can be actively amplified in

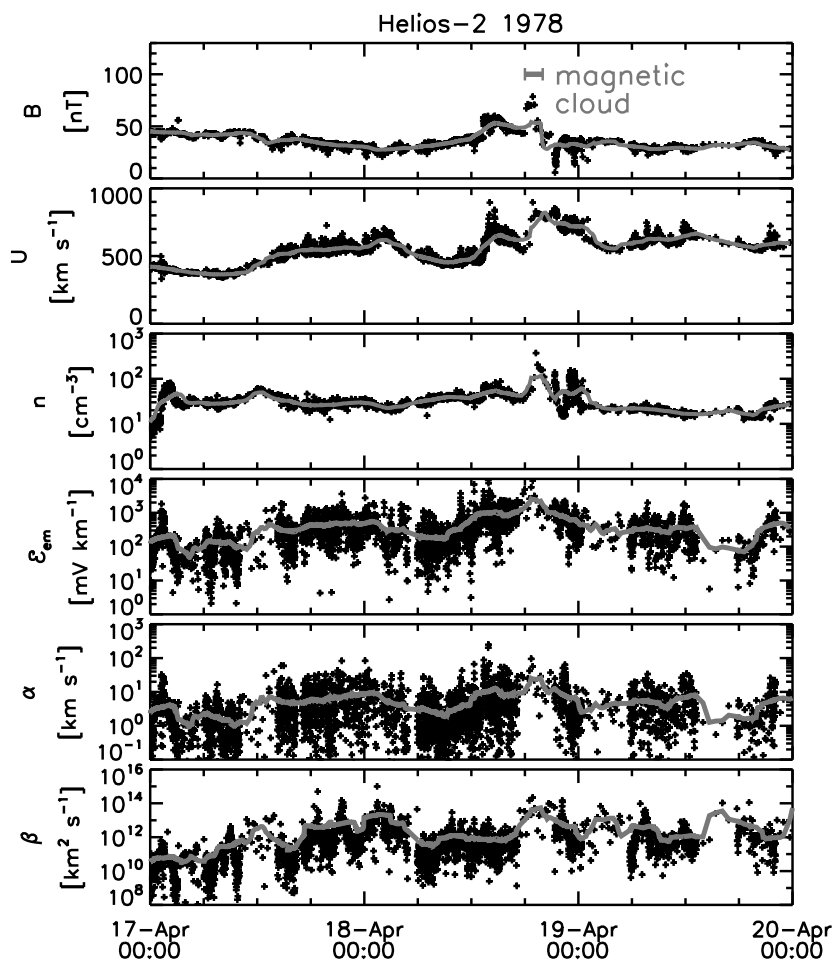

Figure 1. Time series plots of magnetic field magnitude $\boldsymbol{B}$, ion bulk speed $\boldsymbol{U}$, ion density $n$, estimated electromotive force $\mathcal{E}_{\text {em }}$ (in the same units as those of the electric field), coefficients $\alpha$ and $\beta$ for a magnetic cloud event observed by Helios 2 spacecraft in the inner heliosphere from 17 to 20 April 1978.

the heliosphere by the flow shear or twist. We believe, however, that the dynamo action is unlikely to occur in the heliosphere. The enhanced electromotive force or the $\alpha$ effect was associated with the magnetic cloud event for a short time. The electromotive force is largest during the magnetic cloud, but the transport coefficients appear to be large also at other times, notably around the minimum magnetic field. For the magnetic field to grow by a dynamo mechanism, the transport coefficients must stay at larger values for a much longer time period and must also operate on different components (toroidal and poloidal components) of the magnetic field.

There are various ways to improve the method presented here. First, from an accuracy point of view, the regularly sampled data are preferred because the second-order central difference method can be applied to the calculation of the derivatives. Second, for a further evaluation of the mean-field dynamo theory, one may test the relations on the transport coefficients $\alpha=-\frac{1}{3} \tau\langle\delta \boldsymbol{U} \cdot(\nabla \times \delta \boldsymbol{U})\rangle$ (proportional to the kinetic helicity density) and $\beta=\frac{1}{3} \tau\langle\delta \boldsymbol{U} \cdot \delta \boldsymbol{U}\rangle$ (proportional to the kinetic energy density), where $\tau$ denotes the characteristic time of turbulence. To achieve this test, the quantity $\tau$ needs to be determined from the time series data. Third, one may include the cross helicity term in the test for the meanfield dynamo theory. Fourth, the use of multi-point data is 
helpful to relax the assumptions of time stationarity and the alignment of the spatial gradient with the mean flow direction.

Data availability. Helios plasma and magnetic field data are available at CDAWeb https://cdaweb.sci.gsfc.nasa.gov (NASA CDAWeb, 2018).

Competing interests. The authors declare that they have no conflict of interest.

Acknowledgements. This work is financially supported by the Austrian Space Applications Programme (ASAP) at Austrian Research Promotion Agency, FFG ASAP12 SOPHIE, under contract 853994 and Austrian Science Fund (FWF) under contract P28764-N27. Discussion and collaboration with Philippe Bourdin and Bernhard Hofer in the preparation of the manuscript are acknowledged in the framework of the ASAP project. Yasuhito Narita is grateful to Masahiro Hoshino and his group at the University of Tokyo for their hospitality during his research stay, which was supported by the Japan Society for the Promotion of Science, Invitational Fellowship for Research in Japan (short-term) under grant FY2017 S17123.

The topical editor, Georgios Balasis, thanks Octav Marghitu for help in evaluating this paper.

\section{References}

Bruno, R. and Carbone, V.: The solar wind as a turbulence laboratory, Living Rev. Solar Phys., 10, 2, https://doi.org/10.12942/lrsp-2013-2, 2013.

Burch, J. L., Moore, T. E., Torbert, R. B., and Giles, B. L.: Magnetospheric multiscale overview and science objectives, Space Sci. Rev., 199, 5-21, https://doi.org/10.1007/s11214-015-01649, 2016.

Choudhuri, A. R.: The Physics of Fluids and Plasmas: An Introduction for Astrophysicists, Cambridge Univ. Press, Cambridge, 1998.

Escoubet, C. P., Fehringer, M., and Goldstein, M.: Introduction The Cluster mission, Ann. Geophys., 19, 1197-1200, https://doi.org/10.5194/angeo-19-1197-2001, 2001.

Fontana, P. W., Den Hartog, D. J., Fiksel, G., and Prager, S. C.: Spectroscopic observation of fluctuation-induced dynamo in the edge of the reversed-field pinch, Phys. Rev. Lett., 85, 566-569, https://doi.org/10.1103/PhysRevLett.85.566, 2000.

Ji, H. and Prager, S. C.: The $\alpha$ dynamo effects in laboratory plasmas, Magnetohydodyn. 38, 191-210, 2002.

Lundin, R., Sauvaud, J.-A., Rème, H., Balogh, A., Dandouras, I., Bosqued, J. M., Carlson, C., Parks, G. K., Möbius, E., Kistler, L. M., Klecker, B., Amata, E., Formisano, V., Dunlop, M., Eliasson, L., Korth, A., Lavraud, B., and McCarthy, M.: Evidence for impulsive solar wind plasma penetration through the dayside magnetopause, Ann. Geophys., 21, 457-472, https://doi.org/10.5194/angeo-21-457-2003, 2003.
Marsch, E. and Tu, C.-Y.: Electric field fluctuations and possible dynamo effects in the solar wind, Solar Wind Seven, Proceedings of the 3rd COSPAR Colloquium, Goslar, Germany, 16-20 September 1991, edited by: Marsch, E. and Schwenn, R., Pergamonn Press, Oxford, 505-510, https://doi.org/10.1016/B978-008-042049-3.50105-8, 1992.

Marsch, E. and Tu, C.-Y.: MHD turbulence in the solar wind and interplanetary dynamo effects, The Cosmic Dynamo, Proceedings of the 157th Symposium of the International Astronomical Union held in Potsdam, Germany, 7-11 September 1992, edited by: Krause, F., Rädler, K.-H., and Rüdiger, G., International Astronomical Union, Springer, Dordrecht, 51-57, https://doi.org/10.1007/978-94-011-0772-3_9, 1993.

Musmann, G., Neubauer, F. M., Maier, A., and Lammers, E.: Das Förstersonden-Magnetfeldexperiment (E2), Raumfahrtforsch., 19, 232-237, 1975.

Narita, Y.: Review article: Wave analysis methods for space plasma experiment, Nonlin. Processes Geophys., 24, 203-214, https://doi.org/10.5194/npg-24-203-2017, 2017.

NASA CDAWeb: Helios plasma and magnetic field data, available at: https://cdaweb.sci.gsfc.nasa.gov, last access: 23 January 2018.

Parker, E. N.: Hydromagnetic dynamo models, Astrophys. J., 122, 293-314, https://doi.org/10.1086/146087, 1955.

Perschke, C., Narita, Y., Motschmann, U., and Glassmeier, K.H.: Multi-spacecraft observations of linear modes and sideband waves in ion-scale solar wind turbulence, Astrophys. J. Lett., 793, L25, https://doi.org/10.1088/2041-8205/793/2/L25, 2014.

Petrosyan, A., Balogh, A., Goldstein, M. L., Léorat, J., Marsch, E., Petrovay, K., Roberts, B., von Steiger, R., and Vial, J. C.: Turbulence in the solar atmosphere and solar wind, Space Sci. Rev., 156, 135-238, https://doi.org/10.1007/s11214-010-9694-3, 2010.

Porsche, H.: General aspects of the mission Helios 1 and 2, introduction to a special issue on initial scientific results of the Helios mission, J. Geophys., 42, 551-550, 1977.

Rahbarnia, K., Brown, B. P., Clark, M. M., Kaplan, E. T., Nornberg, M. D., Rasmus, A. M., Taylor, N. Z., Forest, C. B., Jenko, F., Limone, A., Pinton, J.-F., Plihon, N., and Verhille, G.: Direct observation of the turbulent emf transport of magnetic field in a liquid sodium experiment, Astrophys. J., 759, 80, https://doi.org/10.1088/0004-637X/759/2/80, 2012.

Rosenbauer, H., Schwenn, R., Marsch, E., Miggenrieder, H., Montgomery, M. D., Mühlhäuser, K. H., Pilipp, W., Voges, W., and Zink, S. M.: A survey on initial results on the Helios plasma experiment, J. Geophys., 42, 561-580, 1977.

Schwenn, R., Rosenbauer, H., and Miggenrieder, H.: Das Plasmaexperiment auf HELIOS (E1), Raumfahrtforsch., 19, 226-232, 1975.

Steenbeck, M. and Krause, F.: Erklärung stellarer und planetarer Magnetfelder durch einen turbubulenzbedingten Dynamomechanismus, Z. Naturforsch., 21a, 1285-1296, https://doi.org/10.1515/zna-1966-0813, 1966.

Steenbeck, M., Krause, F., and Rädler, K.-H.: Berechnung der mittleren Lorentz-Feldstärke $\overline{\boldsymbol{v} \times \boldsymbol{B}}$ für ein elektrisch leitendes Medium in turbulenter, durch Coriolis-Kräfte beeinflußter Bewegung, Z. Naturforsch., 21a, 369-376, https://doi.org/10.1515/zna-1966-0401, 1966. 
Tu, C.-Y. and Marsch, E.: MHD structures, waves, and turbulence in the solar wind: Observations and theories, Space Sci. Rev., 73, 1-210, https://doi.org/10.1007/BF00748891, 1995.

Yokoi, N.: Large-scale magnetic fields in spiral galaxies viewed from the cross-helicity dynamo, Astron. Astrophys., 311, 731745, 1996.

Yokoi, N.: Magnetic-field generation and turbulence suppression due to cross-helicity effects, Phys. Fluids, 11, 2307-2316, https://doi.org/10.1063/1.870093, 1999.
Yokoi, N.: Cross helicity and related dynamo, Geophys. Astrophys. Fluid Dyn., 107, 114-184, https://doi.org/10.1080/03091929.2012.754022, 2013.

Yoshizawa, A.: Self-consistent turbulent dynamo modeling of reversed field pinches and planetary magnetic fields, Phys. Fluids B, 2, 1589-1600, https://doi.org/10.1063/1.859484, 1990.

Yoshizawa, A. and Yokoi, N.: Turbulent magnetohydrodynamic dynamo for accretion disks using the cross-helicity effects, Astrophys. J., 407, 540-548, https://doi.org/10.1086/172535, 1993. 